\title{
A weakly mixing upside-down tower of isometric extensions
}

\author{
S. GLASNER AND B. WEISS † \\ Tel Aviv University and the Hebrew University of Jerusalem
}

(Received 6 June 1980)

\begin{abstract}
On the infinite torus $X=\mathbb{T}^{N}$, using a category argument, we produce a large family of homeomorphisms such that for every element $S$ in this family the flow $(S, X)$ is weakly mixing and strictly ergodic. Moreover, writing $X_{n}=\mathbb{J}^{\{n, n+1 \ldots\}}$ and letting $\pi_{n, m}$, for $n<m$, be the projection of $X_{n}$ on $X_{m}, S$ induces for every $n$, a homeomorphism of $X_{n}$ and the extensions $\left(S, X_{n}\right) \stackrel{\pi_{n, n+1}}{\longrightarrow}\left(S, X_{n+1}\right)$ are isometric. We also show that, for every $S$ in this family, $(S, X)$ is disjoint from every purely weakly mixing flow.
\end{abstract}

\section{Introduction}

For a minimal flow $(T, X)$, where $X$ is a compact metrizable space and $T$ a locally compact group, one defines the notions of distallity and equicontinuity as follows. $(T, X)$ is distal if for some metric $d$ on $X$ (and hence for every metric) $\inf _{t \in T} d\left(t x, t x^{\prime}\right)>0$ for every $x, x^{\prime} \in X$ with $x \neq x^{\prime}$. If for some metric $d$ and every $t \in T, d\left(t x, t x^{\prime}\right)=d\left(x, x^{\prime}\right)$ for all $x, x^{\prime} \in X$, then $(T, X)$ is equicontinuous. Clearly, every equicontinuous flow is distal. That the converse is false was shown by Furstenberg [2] and Auslander, Green and Hahn [1]. We say that $(T, X)$ is an isometric extension of $(T, Y)$ if there exist a homomorphism $(T, X) \stackrel{\pi}{\rightarrow}(T, Y)$ and a continuous function $d$ on $R=\left\{\left(x, x^{\prime}\right) \in X \times X\right.$ : $\left.\pi(x)=\pi\left(x^{\prime}\right)\right\}$ such that:

(i) For every $y \in Y, d$ restricted to $\pi^{-1}(y)$ is a metric.

(ii) $d\left(t x, t x^{\prime}\right)=d\left(x, x^{\prime}\right)$ for all $\left(x, x^{\prime}\right) \in X$ and $t \in T$.

Furstenberg's celebrated structure theorem asserts that with every metrizable distal minimal flow $(T, X)$, there exist a (countable) ordinal $\eta$ and flows $\left(T, X_{\theta}\right)(\theta \leqslant \eta)$, such that:

(1) $X_{0}$ is the trivial one point flow and $X_{\eta}=X$.

(2) For every $\theta<\eta$ there exists an isometric extension

$$
X_{\theta+1} \stackrel{\pi_{\theta}}{\longrightarrow} X_{\theta}
$$

(3) For a limit ordinal $\theta \leq \eta, X_{\theta}=\underset{\beta<\theta}{\operatorname{invlim}} X_{\beta}$.

† Address for correspondence: Dr B. Weiss, Department of Mathematics, Hebrew University of Jerusalem, Jerusalem, Israel. 
The converse of this theorem is clear: namely, every minimal flow possessing such a tower is distal.

A natural question is thus whether a minimal flow which can be represented by an inverted tower of isometric extensions must be distal? More precisely, suppose that $(T, X)$ is a minimal flow; suppose further that, for every positive integer $n$, there exist a flow $X_{n}$ and a homomorphism $X_{n} \stackrel{\pi_{n}}{\longrightarrow} X_{n+1}$ such that:

(1') $X_{1}=X$.

(2') $\pi_{n}$ is an isometric extension for every $n$.

(3') The largest common factor of all the $X_{n}$ is the trivial flow.

(Equivalently, the set

$$
\left\{\left(x, x^{\prime}\right) \in X \times X: \exists n \pi_{n} \circ \cdots \circ \pi_{1}(x)=\pi_{n} \circ \cdots \circ \pi_{1}\left(x^{\prime}\right)\right\}
$$

is dense in $X \times X$.) Is $(T, X)$ necessarily distal?

In this note we show that the answer to our question is negative. We construct a large family of minimal flows on the infinite torus, satisfying properties $\left(1^{\prime}\right)-\left(3^{\prime}\right)$, which are non-distal. In fact, they are weakly mixing with respect to the usual product measure and hence also topologically weakly mixing. The latter property implies that these flows are actually disjoint from every minimal distal flow (recall that two minimal flows are disjoint if their product is minimal).

The easiest way of characterizing pure weak mixing is as follows. A minimal flow $(T, X)$ is purely weak mixing (p.w.m.) if, for every topologically weakly mixing minimal flow $(T, Y)$, every minimal subset of $(T, X \times Y)$ is topologically weakly mixing. This is not the original definition of p.w.m. which is rather technical [3]. It follows immediately from [3] that a minimal flow satisfying $\left(1^{\prime}\right)-\left(3^{\prime}\right)$ above, cannot be p.w.m. and, in fact, we shall show that such a flow is disjoint from every p.w.m. flow. The first author is indebted to Prof. R. Ellis for pointing this out. Of course this falsifies the conjecture that every topologically weakly mixing, non-trivial, minimal flow possesses a non-trivial p.w.m. factor.

Our method of construction is that of [5]. We refer the reader to [4] for more details on the notions mentioned in this introduction.

\section{The set $\overline{\mathscr{S}}$}

We let $\mathbb{Z}$ be the set of integers, $\mathbb{N}$ the set of positive integers, $\mathbb{R}$ the real numbers and $\mathbb{V}=\mathbb{R} / \mathbb{Z}$ the additive group of reals modulo one. Let $|\cdot|$ stand for the distance of a real number from the closest integer. Put $X=T^{N} ; X$ is a compact monothetic group and we choose an element $\alpha=\left(\alpha_{1}, \alpha_{2}, \alpha_{3}, \ldots\right)$ in $X$ such that the set $\{n \alpha\}_{n=-\infty}^{\infty}$ is dense in $X$. Let $\sigma: X \rightarrow X$ be the homeomorphism of $X$ defined by $\sigma x=x+\alpha$. We write $d x$ for Lebesgue measure on $T$ and $\mu$ will be the corresponding product measure on $X$. Clearly $\mu$ is invariant under $\sigma$. With every finite sequence of continuous functions $\left\{f_{i}\right\}_{i=1}^{n}, f_{i}: \mathbb{T} \rightarrow \mathbb{T}$, we associate a homeomorphism $F$ of $X$ as follows:

$F\left(x_{1}, x_{2}, \ldots, x_{n}, x_{n+1}, \ldots\right)=\left(x_{1}+f_{1}\left(x_{2}\right), x_{2}+f_{2}\left(x_{3}\right), \ldots, x_{n}+f_{n}\left(x_{n+1}\right), x_{n+1}, \ldots\right)$.

Let $\mathscr{F}$ be the family of all the homeomorphisms of $X$ obtained in this way and let

$$
\mathscr{S}=\mathscr{S}(\sigma)=\left\{F^{-1} \circ \sigma \circ F: F \in \mathscr{F}\right\} .
$$


Notice that if $S=F^{-1} \circ \sigma \circ F$ where $F$ is as above then

$$
\begin{aligned}
S\left(x_{1}, x_{2}, \ldots\right)= & \left(x_{1}+\alpha_{1}+f_{1}\left(x_{2}\right)-f_{1}\left(x_{2}+\alpha_{2}+f_{2}\left(x_{3}\right)-\cdots\right.\right. \\
& \left.-f_{n-1}\left(x_{n}+\alpha_{n}+f_{n}\left(x_{n+1}\right)-f_{n}\left(x_{n+1}+\alpha_{n+1}\right)\right) \cdots\right), \\
& x_{2}+\alpha_{2}+f_{2}\left(x_{3}\right)-f_{2}\left(x_{3}+\alpha_{3}+f_{3}\left(x_{4}\right)-\cdots\right. \\
& \left.-f_{n-1}\left(x_{n}+\alpha_{n}+f_{n}\left(x_{n+1}\right)-f_{n}\left(x_{n+1}+\alpha_{n+1}\right)\right) \cdots\right), \\
& \vdots \\
& \left.x_{n}+\alpha_{n}+f_{n}\left(x_{n+1}\right)-f_{n}\left(x_{n+1}+\alpha_{n+1}\right), x_{n+1}+\alpha_{n+1}, x_{n+2}+\alpha_{n+2}, \ldots\right) .
\end{aligned}
$$

We consider $\mathscr{S}(\sigma)$ as a subset of the space of all homeomorphisms of $X$ with the topology of uniform convergence of homeomorphisms and their inverses. $\overline{\mathscr{S}}(\sigma)=\overline{\mathscr{S}}$ will be the closure of $\mathscr{S}$ in this space.

Since each $F \in \mathscr{F}$ preserves $\mu$ so do all the homeomorphisms in $\overline{\mathscr{S}}$.

For every $n \in \mathbb{N}$ let $X_{n}=\mathbb{T}^{\{n, n+1, \ldots\}}$ and for $n<m$ let $\pi_{n, m}$ be the projection of $X_{n}$ onto $\boldsymbol{X}_{m}$. Write $\pi_{n}$ for $\pi_{1, n}$.

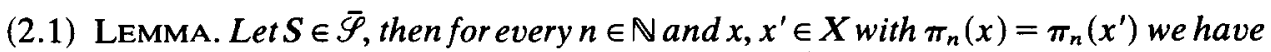
$\pi_{n}(S x)=\pi_{n}\left(S x^{\prime}\right)$. Thus $S$ induces a homeomorphism $S_{n}$ of $X_{n}$ and $(S, X) \stackrel{\pi_{n}}{\longrightarrow}\left(S_{n}, X_{n}\right)$ is a flow homomorphism. Moreover, for every $n,\left(S_{n}, X_{n}\right) \stackrel{\pi_{n, n+1}}{\longrightarrow}\left(S_{n+1}, X_{n+1}\right)$ is a circle (hence an isometric) extension. The largest common factor of the flows $\left(S_{n}, X_{n}\right), n=1,2, \ldots$, is the trivial flow.

Proof. Let $S \in \mathscr{S}$, then $S$ has the form (*) for some finite sequence $\left\{f_{i}\right\}_{i=1}^{n}$. By considering (*), it is clear that the first statement of the lemma holds for $S$.

Since $\mathscr{S}$ is dense in $\overline{\mathscr{S}}$ it also holds for every element of $\overline{\mathscr{S}}$. For $S \in \overline{\mathscr{S}}$ and $n \in \mathbb{N}$ we now define $S_{n}$ by $S_{n}\left(\pi_{n}(x)\right)=\pi_{n}(S x)$. We leave to the reader the easy verification of the remaining statements of the lemma.

\section{The sets $U_{\phi}(\eta)$}

Let $C(X)$ be the space of bounded continuous complex-valued functions on $X$ with sup norm $\|\cdot\|$.

$$
C_{1}(X)=\left\{f \in C(X): \int f d \mu=0 \quad \text { and } \int|f|^{2} d \mu=1\right\} .
$$

For elements $\phi, \psi \in L_{2}(\mu)$ we write

$$
\langle\phi, \psi\rangle=\int \phi \bar{\psi} d \mu \text { and }\|\phi\|_{2}=\langle\phi, \phi\rangle^{1 / 2} \text {. }
$$

If $T$ is a measure preserving homeomorphism of $X$ and $\phi \in L_{2}(\mu)$ then $T \phi \in L_{2}(\mu)$ is defined by $(T \phi)(x)=\phi(T x)$.

(3.1) LEMMA. Let $T$ be a measure preserving homeomorphism of $X$. Then $T$ is weakly mixing iff there exists a subset $\left\{\phi_{i}\right\}_{i=1}^{\infty}$ of $C_{1}(X)$ which is dense in $C_{1}(X)$ and such that $\forall i \exists n$ with $\left|\left\langle T^{n} \phi_{i}, \phi_{i}\right\rangle\right| \leq 0.99$.

Proof. This follows easily from the fact that $T$ is weakly mixing iff $\forall \phi \in L_{2}(\mu)$

$$
\frac{1}{n+1} \sum_{k=0}^{n}\left|\left\langle T^{k} \phi, \phi\right\rangle-\langle\phi, 1\rangle\langle 1, \phi\rangle\right| \rightarrow 0
$$

iff $T$ has a continuous spectrum on $L_{2}(\mu)$. 
For $\phi \in C_{1}$ and $0<\eta<1$ let

$K_{\phi}(\eta)=\{T: T$ is a measure preserving homeomorphism of $X$ and $\exists n$ with $\left.\left|\left\langle T^{n} \phi, \phi\right\rangle\right| \leq \eta\right\}$,

and

$$
U_{\phi}(\eta)=K_{\phi}(\eta) \cap \overline{\mathscr{S}} \text {. }
$$

(3.2) LEMMA. Let $\phi(x)=\sum c_{n_{1} \ldots n_{k}} \exp \left[2 \pi i\left(n_{1} x_{1}+\cdots+n_{k} x_{k}\right)\right]$ be an element of $L_{2}(\mu)$ with $\|\phi\|_{2}=1$ i.e. with $\sum\left|c_{n_{1}} \ldots n_{k}\right|^{2}=1$. Suppose that there exists an $\eta, 0<\eta<1$, such that for every index $\left(n_{1}, \ldots, n_{k}\right) \in \mathbb{Z}^{k},\left|c_{n_{1}} \ldots n_{k}\right| \leq \eta$; then $\sigma \in U_{\phi}(\eta)$.

Proof. Let $p_{n_{1} \ldots n_{k}}=\left|c_{n_{1} \ldots n_{k}}\right|^{2}$ and let $\nu$ be the probability measure on $\mathbb{T}$ defined by

For every $j \in \mathbb{Z}$

$$
\nu=\sum p_{n_{1} \ldots n_{k}} \delta_{n_{1} \alpha_{1}+\cdots+n_{k} \alpha_{k}} \text {. }
$$

$$
\begin{aligned}
\left|\left\langle\sigma^{j} \phi, \phi\right\rangle\right|= & \left|\int \phi\left(\sigma^{i} x\right) \overline{\phi(x)} d \mu(x)\right| \\
= & \mid \sum c_{n_{1} \cdots n_{k}} \bar{c}_{m_{1} \cdots m_{l}} \int \cdots \int \exp \left[2 \pi i\left(n_{1}\left(x_{1}+j \alpha_{1}\right)+\cdots+n_{k}\left(x_{k}+j \alpha_{k}\right)\right)\right] \\
& \exp \left[2 \pi i\left(-m_{1} x_{1}-\cdots-m_{l} x_{l}\right)\right] d x_{1} \cdots d x_{\max (k, l)} \mid \\
= & \left.\left|\sum\right| c_{n_{1} \cdots n_{k}}\right|^{2} \exp \left[2 \pi i j\left(n_{1} \alpha_{1}+\cdots+n_{k} \alpha_{k}\right)\right] \mid \\
= & \left|\int_{T} \exp [2 \pi i j x] d \nu(x)\right|=|\hat{\nu}(j)| .
\end{aligned}
$$

By Wiener's theorem

$$
\lim \frac{1}{2 N+1} \sum_{-N}^{N}|\hat{\nu}(j)|^{2}=\sum p_{n_{1} \cdots n_{k}}^{2}=\sum\left|c_{n_{1} \cdots n_{k}}\right|^{4} \leq \eta^{2} \sum p_{n_{1} \cdots n_{k}}=\eta^{2} .
$$

Thus for some $n,|\hat{\nu}(n)|^{2}=\left|\left\langle\sigma^{n} \phi, \phi\right\rangle\right|^{2} \leq \eta^{2}$ and $\left|\left\langle\sigma^{n} \phi, \phi\right\rangle\right| \leq \eta$.

(3.3) LEMMA. Let $F \in \mathscr{F}, \phi \in C_{1}$ then $F^{-1} K_{\phi}(\eta) F=K_{\phi \circ F}(\eta)$.

Proof. For $S \in K_{\phi}$ we have

$$
\begin{aligned}
\left\langle F S^{n} F^{-1} \phi, \phi\right\rangle & =\int \phi\left(F S^{n} F^{-1} x\right) \overline{\phi(x)} d \mu(x)=\int \phi\left(F S^{n} x\right) \overline{\phi(F x)} d \mu(x) \\
& =\left\langle S^{n}(\phi \circ F), \phi \circ F\right\rangle .
\end{aligned}
$$

Therefore

$$
\begin{aligned}
F^{-1} K_{\phi}(\eta) F= & \{S: S \text { is a measure preserving homeomorphism of } X \text { and } \\
& \left.\exists n,\left|\left\langle F S^{n} F^{-1} \phi, \phi\right\rangle\right| \leq \eta\right\} \\
= & K_{\phi \circ F}(\eta) .
\end{aligned}
$$

(3.4) LEMMA. Let $g$ be a continuous real-valued function on $\mathbb{T}$ which is twice differentiable and assume that $g^{\prime \prime}$ has only finitely many zeros, then

$$
\lim _{|n| \rightarrow \infty}\left\{\sup _{m}\left|\int \exp [2 \pi i(n g(x)-m x)] d x\right|\right\}=0 .
$$


Proof. This follows easily from van-der-Corput's lemma; see, for example [6, p. 220].

\section{The main theorem}

(4.1) TheOREM. There exists a dense $G_{\delta}$ subset, $\mathscr{R}$ of $\overline{\mathscr{S}}$ such that for every $S \in \mathscr{R}$, $(S, X)$ is strictly ergodic (hence minimal) and weakly mixing.

Proof. Since $\sigma$ is strictly ergodic on $X$ so is every homeomorphism of $X$ of the form $F^{-1} \circ \sigma \circ F(F \in \mathscr{F})$. Thus for every $f \in C(X)$ and $\varepsilon>0$ the set

$$
E_{f, \varepsilon}=\left\{S \in \overline{\mathscr{S}}: \exists n \text { and } c \text { with }\left\|\frac{1}{n+1} \sum_{k=0}^{n} f\left(S^{k} x\right)-c\right\|<\varepsilon\right\},
$$

which is clearly open, is also dense in $\overline{\mathscr{S}}$. Put $\mathscr{R}_{1}=\bigcap_{n=1}^{\infty} \bigcap_{i=1}^{\infty} E_{f_{i}, 1 / n}$, where $\left\{f_{i}\right\}_{i=1}^{\infty}$ is a dense subset of $C(X)$. Then $\mathscr{R}_{1}$ is a dense $G_{\delta}$ subset of $\overline{\mathscr{S}}$ which consists of strictly ergodic homeomorphisms.

We shall show next that for every $\phi \in C_{1}, U_{\phi}(0.99)$ is an open and dense subset of $\overline{\mathscr{S}}$. If $\left\{\phi_{i}\right\}_{i=1}^{\infty}$ is a dense subset of $C_{1}$ then, by lemma 3.1 , every homeomorphism in $\mathscr{R}_{2}=\bigcap_{i=1}^{\infty} U_{\phi_{i}}(0.99)$ is weakly mixing and we can put $\mathscr{R}=\mathscr{R}_{1} \cap \mathscr{R}_{2}$.

So let $\phi \in C_{1}$; clearly $U_{\phi}(0.99)$ is open. To see that it is dense it suffices to show that, for every $F \in \mathscr{F}$,

$$
F^{-1} \circ \sigma \circ F \in \overline{K_{\phi}(0.99) \cap \mathscr{P}} \subset \overline{K_{\phi}(0.99) \cap \overline{\mathscr{S}}}=\overline{U_{\phi}(0.99)} .
$$

If $\left\{G_{n}\right\}_{n=1}^{\infty} \subset \mathscr{F}$ is a sequence such that $G_{n} \circ \sigma \circ G_{n}^{-1} \rightarrow \sigma$ and such that $\forall n G_{n} \circ \sigma \circ G_{n}^{-1} \in K_{\phi \circ F}(0.99)$ then clearly

$$
F \circ \sigma \circ F^{-1}=\lim _{n} F \circ G_{n} \circ \sigma \circ G_{n}^{-1} \circ F^{-1} \in \overline{K_{\phi}(0.99) \cap \mathscr{S}} \quad \text { (lemma 3.3). }
$$

Thus it is enough to prove the following

(4.2) LEMMA. Let $\varepsilon>0$, then there exists $G \in \mathscr{F}$ with

(i) $d\left(G \circ \sigma \circ G^{-1}, \sigma\right)<\varepsilon$.

(ii) $G \circ \sigma \circ G^{-1} \in K_{\phi}(0.99)$ i.e. $\sigma \in K_{\phi \circ G}(0.99)$.

Proof. Let $\phi(x)=\sum c_{n_{1} \ldots n_{k}} \exp \left[2 \pi i\left(n_{1} x_{1}+\cdots+n_{k} x_{k}\right)\right]$ be the Fourier expansion of $\phi$ in $L_{2}(\mu)$. If for every $\left(n_{1}, \ldots, n_{k}\right),\left|c_{n_{1} \ldots n_{k}}\right| \leq 0.99$, by lemma 3.2 , we have $\sigma$ $\in K_{\phi}(0.99)$ and'we can take $G=i d$. Otherwise, there is exactly one index $\left(n_{1}, \ldots, n_{k}\right)$ such that $\left|c_{n_{1} \ldots n_{k}}\right|>0.99$. Denote $c_{n_{1} \ldots n_{k}}$ by $c_{0}$ and let $\phi_{1}=c_{0} \exp \left(n_{1} x_{1}+\cdots+n_{k} x_{k}\right)$ and $\phi_{2}=\phi-\phi_{1}$. Then $\phi_{1}, \phi_{2} \in L_{2}(\mu)$ and $1=\|\phi\|_{2}^{2}=\left\|\phi_{1}\right\|_{2}^{2}+\left\|\phi_{2}\right\|_{2}^{2}$. Since $\left\|\phi_{1}\right\|_{2}^{2}$ $=\left|c_{0}\right|^{2}>0.9801,\left\|\phi_{2}\right\|_{2}^{2} \leq 0.02$ and $\left\|\phi_{2}\right\|_{2} \leq 0.2$. Now, for every $n$,

$$
\begin{aligned}
\left|\left\langle\sigma^{n}(\phi \circ G), \phi \circ G\right\rangle\right| \leq & \left|\left\langle\sigma^{n}\left(\phi_{1} \circ G\right), \phi_{1} \circ G\right)\right|+\left|\left\langle\sigma^{n}\left(\phi_{2} \circ G\right), \phi_{2} \circ G\right\rangle\right| \\
& +\left|\left\langle\sigma^{n}\left(\phi_{1} \circ G\right), \phi_{2} \circ G\right)\right|+\left|\left\langle\sigma^{n}\left(\phi_{2} \circ G\right), \phi_{1} \circ G\right\rangle\right| \\
\leq & \left|\left\langle\sigma^{n}\left(\phi_{1} \circ G\right), \phi_{1} \circ G\right)\right|+3\left\|\phi_{2}\right\|_{2} .
\end{aligned}
$$

Thus in order to establish (ii) it is enough to find $G \in \mathscr{F}$ and $n$ for which $\left|\left\langle\sigma^{n}\left(\phi_{1} \circ G\right), \phi_{1} \cdot G\right\rangle\right| \leq 0.3$; or putting $\phi_{0}=\phi_{1} / c_{0}$ - since $\left|c_{0}\right| \leq 1$ - it suffices to show that $\left|\left\langle\sigma^{n}\left(\phi_{0} \circ G\right), \phi_{0} \circ G\right\rangle\right| \leq 0.3$, i.e. that $\sigma \in K_{\phi_{0} \circ G}(0.3)$. 
But $\phi_{0}$ and hence also $\psi=\phi_{0} \circ G$ are in $C_{1}$ and, by lemma -3.2 , it suffices to find $G \in \mathscr{F}$ such that for every $\left(m_{1}, \ldots, m_{l}\right)$

$$
\left|\left\langle\phi_{0} \circ G, \exp \left[2 \pi i\left(m_{1} x_{1}+\cdots+m_{l} x_{l}\right)\right]\right\rangle\right| \leq 0.3 .
$$

Let $G \in \mathscr{F}$ have the form

$$
G\left(x_{1}, \ldots, x_{k}, \ldots\right)=\left(x_{1}, \ldots, x_{k-1}, x_{k}+f\left(x_{k+1}\right), x_{k+1}, \ldots\right) .
$$

Then, for every $\left(m_{1}, \ldots, m_{l}\right)$

$$
\begin{aligned}
\mid\left\langle\phi_{0}\right. & \left.\circ G, \exp \left[2 \pi i\left(m_{1} x_{1}+\cdots+m_{l} x_{l}\right)\right]\right) \mid \\
\quad & \left.\exp \left[2 \pi i\left(n_{1} x_{1}+\cdots+n_{k} x_{k}+n_{k} f\left(x_{k+1}\right)\right)\right], \exp \left[2 \pi i\left(m_{1} x_{1}+\cdots+m_{l} x_{l}\right)\right]\right\rangle \mid \\
\quad & =\delta_{0}\left|\int \exp \left[2 \pi i\left(n_{k} f\left(x_{k+1}\right)-m_{k+1} x_{k+1}\right)\right] d x_{k+1}\right|,
\end{aligned}
$$

where $\delta_{0}$ is either 0 or 1 .

Let $g: \mathbb{T} \rightarrow \mathbb{R}$ be twice differentiable and such that $g^{\prime \prime}$ has only finitely many zeros. Then, by lemma 3.4, we shall have, for some $n_{0} \in \mathbb{Z}$,

$$
\left|\int \exp \left[2 \pi i\left(n_{k} n_{0} g(x)-m_{k+1} x\right)\right] d x\right| \leq 0.3
$$

Finally, let $\delta>0$ be such that $\left|x-x^{\prime}\right|<\delta \Rightarrow\left|n_{0} g(x)-n_{0} g\left(x^{\prime}\right)\right|<\varepsilon$ and let $q \in \mathbb{Z}$ be such that $\left|q \alpha_{k+1}\right|<\delta$. Define $f(x)=n_{0} g(q x)$ : then for every $x \in \mathbb{T}\left|f\left(x+a_{k+1}\right)-f(x)\right|<\varepsilon$ and for

$$
G\left(x_{1}, \ldots, x_{k}, \ldots\right)=\left(x_{1}, \ldots, x_{k-1}, x_{k}+f\left(x_{k+1}\right), x_{k+1}, \ldots\right)
$$

we have

$$
\begin{aligned}
G^{-1} \circ \sigma \circ G\left(x_{1}, \ldots, x_{k}, \ldots\right)= & \left(x_{1}+\alpha_{1}, \ldots, x_{k-1}+\alpha_{k-1}, x_{k}+f\left(x_{k+1}\right)+\alpha_{k}\right. \\
& \left.-f\left(x_{k+1}+\alpha_{k+1}\right), x_{k+1}+\alpha_{k+1}, \ldots\right)
\end{aligned}
$$

and $d\left(\sigma, G^{-1} \circ \sigma \circ G\right)<\varepsilon$.

The set of non-zero Fourier coefficients of $\exp \left[2 \pi i n_{k} n_{0} g(x)\right]$ is the same as that of $\exp \left[2 \pi i n_{k} f(x)\right]$ and the proof of the lemma is complete. This also completes the proof of theorem 4.1.

\section{Disjointness}

Let $u$ be an idempotent in a minimal ideal $M$ of $\beta \mathbb{Z}$. Let $S \in \mathscr{R}$ (see theorem 4.1) and let $x_{0}$ be a point of the flow $(S, X)$ with $u x_{0}=x_{0}$. Write $A=A_{1}=\mathscr{J}\left(S, X, x_{0}\right)$, the Ellis group of the pointed flow $\left(S, X, x_{0}\right)$. For $n \in \mathbb{N}$ let $A_{n}=\mathscr{J}\left(S_{n}, X_{n}, \pi_{n}\left(x_{0}\right)\right)$. Notice that the fact that the largest common factor of the flows $\left(S_{n}, X_{n}\right)$ is the trivial flow implies that $\bigcup A_{n}$ is $\tau$-dense in $G=u M$. We let $P$ denote the Ellis group of the universal p.w.m. $\mathbb{Z}$-flow.

Since, for each $n, X_{n}$ is an isometric extension (and hence an almost periodic extension) of $X_{n+1}$ we have

$$
H\left(A_{n+1}\right) \subset A_{n} \quad[5, \text { theorem IX, 2.1(4)]. }
$$

Since $P$ is p.w.m. we have, for each $n$,

$$
H\left(A_{n+1}\right)\left(P \cap A_{n+1}\right)=A_{n+1} \text {. }
$$


Thus $A_{n}\left(P \cap A_{n+1}\right)=A_{n+1}$ and $A_{n} P \supset A_{n+1}$. Now $A_{n} P=A_{n} P P \supset A_{n+1} P=$ $A_{n+1} P P \supset A_{n+2} P$ and, by induction, we have for every $n, A P \supset A_{n}$. Since cl s $v_{\tau} \cup A_{n}=$ $G$ we conclude that $A P=G$. This, for $\mathbb{Z}$-flows, implies disjointness and we have proved the following theorem.

(5.1) THEOREM. For every element $S \in \mathscr{R}$, the weakly mixing minimal flow $(S, X)$ is disjoint from every p.w.m. flow.

\section{REFERENCES}

[1] L. Auslander, L. Green \& F. Hahn. Flows on Homogeneous Spaces. Ann. of Math. Studies no. 53. Princeton University Press: Princeton, 1963.

[2] H. Furstenberg. The structure of distal flows. Amer. J. Math. 85 (1963), 477-515.

[3] R. Ellis \& S. Glasner. Pure weak mixing. T.A.M.S. 243 (1978), 135-146.

[4] S. Glasner. Proximal Flows. Springer Lecture Notes in Math. no. 517. Springer: Berlin, 1976.

[5] S. Glasner \& B. Weiss. On the construction of minimal skew products. Israel J. Math. (to appear).

[6] Y. Katznelson. An Introduction to Harmonic Analysis. Wiley: New York, 1968. 